Background A growing emphasis on patient involvement in health care decisions has brought 'patient participation' and 'informed choice' to the core of the debates. Health care decisions, based on interpersonal relationships between the health worker and the patient, may be viewed along a continuum from paternalistic approaches to approaches emphasising counselling, patient involvement and patient choice. A key question is how the new models are implemented and experienced in diverging cultural contexts. Infant feeding by HIV positive women in low income contexts is challenging in terms of health care decisions, and rapidly changing guidelines complicate the picture. The present study explored experiences with different approaches to health care decisions within infant feeding counselling in 'prevention of mother-to-child transmission of HIV' (PMTCT) programmes.

Methods 9 months of ethnographic fieldwork were conducted in two hospitals in rural and semi-urban areas of Tanzania. Core informants were nurses and HIV positive women.

Results Hospital A presented no informed patient choice in infant feeding counselling - emphasising exclusive breastfeeding. The programme adapted their approach to what seemed realistic in terms of conditions of little or no actual choices. HIV positive women in hospital A communicated a clear understanding of the 'instructions' given. Hospital B expressed that they followed the national guidelines emphasising patient choice. The women enrolled in this programme experienced confusing messages. Findings show that in none of the hospitals counselling and choice principles were implemented as presented in the literature, as they were not found feasible.

Conclusion In health care systems which have been dominated by paternalistic interactions, partly due to the knowledge gap between the health workers and patients, the introduction of concepts such as counselling and patient choice are challenged both by culturally constituted notions of decision making and by patient groups with limited knowledge and with no actual feeding choices.

\section{P4.143 THE RELATIONSHIP BETWEEN EMOTIONAL INTELLIGENCE AND MENTAL HEALTH WITH HIV-RELATED HIGH RISK BEHAVIOURS AMONG FEMALE SEX WORKERS IN SHIRAZ - IRAN}

doi:10.1136/sextrans-2013-051184.1039

'M Amini Lari, $2 \mathrm{P}$ Bagheri, ${ }^{3} \mathrm{~F}$ Ameli. 'Shiraz HIVIAIDS Research Center, Shiraz University of Medical Science, Shiraz, Iran; ${ }^{2}$ Social Development and Health Promotion Research Center, Gonabad University of Medical Sciences, Gonabad, Iran; ${ }^{3}$ Zand High Education Institute, Shiraz, Iran

Background Psychological problems have significant associations with HIV-related high-risk behaviours among female sex workers (FSW). The present study aimed to investigate emotional intelligence and mental health and their relationships with high-risk behaviours among FSW in Shiraz, Iran.

Methods This cross-sectional study, conducted from October 2011 to May 2012, was based on a convenience sampling method used to recruit $125 \mathrm{FSW}$ and 125 control women. After providing informed consent, each woman completed validated questionnaires (SCL90-R, Bar-On Emotional Quotient [EQ] Inventory and high-risk behaviours).

Results In comparison to the control group, the mean age at first sexual relationship was lower among sex workers, and most of them $(80.4 \%)$ had not used condoms in their most recent sexual intercourse. FSW showed lower EQ scores and more psychological symptoms in all dimensions $(\mathrm{P}<0.003)$. A significant inverse relationship was found between global severity indexes (GSR) scores and use of a condom in the most recent sexual intercourse. According to EQ scores, significant relationships were observed for interpersonal skills and stress management with the number of sexual partners during the previous week and condom use in the most recent sexual intercourse $(\mathrm{P}<0.05)$.

Discussion Considering the relationship of high-risk behaviours with lower emotional intelligence and mental health among FSW, psychological interventions are needed in order to prevent high-risk behaviours and HIV transmission.

\section{P4.144 WITHDRAWN BY AUTHOR}

\section{P4.145 HEALTH PROMOTION STRATEGIES FOR DEVELOPING LIFE SKILLS TO FIGHT AGAINST HIV/AIDS AMONG SCHOOL CHILDREN}

doi:10.1136/sextrans-2013-051184.1040

J P h M Vidanapathirana, S Nanayakkara, G Samaraweera. National STD/AIDS Control Programme, COLOMBO 10, Sri Lanka

Introduction Sri Lankan schools setup makes every effort to improve wellbeing of school children. One objective is develop life skills to promote sexual well being. Advocacy, skill building of education officer and school teachers were carried out using health promotion strategies with participatory approach. Health promoting schools were established and concept were introduced to achieve in which the school community for developing life skills.

Objective To assess the usefulness of Health promotion strategies for developing life skills to fight against HIV/AIDS among school children

Methodology Audit and check list methods were used to assess the application of some health promotion strategies. Altogether 20 pre and post focus group discussions (FGDs) were conducted, with school teachers before and after establishing the health promoting schools to assess the knowledge on HIV/AIDS. Content analysis was used. A quantitative post intervention survey were carried out to assess knowledge and attitudes among school children using a scientific sampling technique in three consecutive years

Results Audit and check list methods revealed that more than $80 \%$ of schools were implemented health promotion strategies in related to HIV/AIDS prevention by adopting various strategies including the relevant policies.

FGDs revealed that the majority of teachers had poor knowledge on transmission and prevention of HIV/AIDS and post intervention FGDs revealed that majority had improved the knowledge up to satisfactory level on transmission and prevention.

Quantitative survey among school children showed more than $80 \%$ of school children ranked the knowledge on transmission, prevention and misconception of HIV/AIDS during year 2009 and $90 \%$ and $89 \%$ in year 2010and 2012 respectively.

Conclusions Skill based education via school teachers is a successful method to improve knowledge and attitude among school children on HIV prevention. Health promotion strategies were useful for developing life skills to fight against HIV/AIDS among school children

\section{P4.146 DISCLOSING GENITAL HERPES: PERCEPTIONS OF MOTIVATION, LOGISTICS AND TIMING}

doi:10.1136/sextrans-2013-051184.1041

1,2M Catallozzi, 'S C Ebel, 'N R Chavéz, 'L Shearer, ${ }^{3} \mathrm{~A}$ Mindel, 'S L Rosenthal 'Columbia University College of Physicians \& Surgeons, New York, NY, United States, ${ }^{2}$ Columbia University Mailman School of Public Health, New York, NY, United States, ${ }^{3}$ University of Sydney School of Medical, Sydney, Australia

Objective The aim of the study was to evaluate the lay public's portrayal of disclosure of Herpes simplex virus (HSV) infection. Methods The Australian Herpes Management Forum and Novartis Pharmaceuticals organised an on-line contest in 2011, challenging 
contestants to develop 30-second videos addressing 'how you tell a partner you have $H S V^{\prime}$ ' given that 'for many people shame and stigma are so overwhelming that they don't feel comfortable talking about it.'

Results Sixty-three videos were analysed for the following thematic content regarding disclosure: motivation, strategies/logistics and timing/context. The portrayed motivations included: no reason not to disclose, given the ease of disease management; to be consistent with values (e.g., respect, honesty); or to avoid the risk of accidental 'outing'. Other videos only commanded disclosure without providing a rationale. With regards to strategies and logistics, most disclosures occurred in a private or semi-private setting, often of a romantic/intimate nature and in a direct manner. However, some used an indirect/ non-verbal approach (e.g., giving a pamphlet or as part of a game). A few showed consideration of the partner's need for time to respond. A few showed the individual practising disclosure. The timing and context of disclosure varied and was often unclear; however, some videos demonstrated disclosure occurring post-coitally.

Conclusions Some video creators viewed disclosure as occurring in romantic settings, post-coitally, and in a non-direct manner, all of which are not consistent with traditional counselling messages. Disclosing in a romantic setting and in non-direct ways may be reasonable; it would still be desirable for disclosure to occur pre-coitally. Understanding these differences may help develop counselling messages that resonate with patient expectations and are more effective in promoting disclosure. Future research should collect patient experiences and perceived outcomes of disclosure in terms of the setting, method, and timing of disclosure.

\section{P4.147 'NO RESPECT, NO GOOD SEX': SEXUAL ETHICS IN BAREBACKING PRACTICES}

doi:10.1136/sextrans-2013-051184.1042

E Daroya. Australian Research Centre in Sex, Health \& Society, La Trobe University, Melbourne, Australia

Research on 'bugchasing' or intentional seroconversion generally focuses on the psychosocial motivations for why gay and other homosexually active men engage in such practises, but fails to attend to the question of ethics. Conversely, studies on barebacking rarely consider how sexual ethics are shaped and entangled with other knowledge practises. This paper explores how issues of responsibility and consent (as sexual ethics) are conceptualised and practised in an online forum on barebacking. In particular, I focus on 'stealthing': broadly defined as barebacking practise involving the deliberate non-disclosure of HIV-status to intentionally infect or become infected with HIV. Using online ethnography, I explore the relational ties between biomedical practises (e.g. testing regimes, knowledge of viral load, etc), legal apparatuses and normative ethics that frame ethical debates on stealthing, bugchasing and barebacking. I argue that men in the forum construct responsibility as polysemic: some men uphold self-protection; some believe HIV-positive men must take responsibility to protect their partners; while others emphasise shared responsibility by appealing to ideas of community. However, they almost universally draw on notions of consent and choice shaping barebacking and bugchasing as consensual, while regarding stealthing as morally unacceptable. This study aims to attend to the complex and multiple processes that shape decision making in regard to unsafe sexual practises.

\section{P4.148 SEXUAL PRACTISE AND HIV IN UGANDA: THE SEARCH FOR "LIVE SEX"}

doi:10.1136/sextrans-2013-051184.1043

1.2S K H Mylan. ' ${ }^{2}$ Southend University Hospital, Southend on Sea, UK; ${ }^{2}$ Brunel University, London, UK
Background Across Sub-Saharan Africa, HIV is still predominantly spread via heterosexual intercourse. Understanding sexuality in this region and its relationship with HIV is, therefore, a vital aspect of understanding the disease. Anthropology has offered many perspectives trying to better understand the social and cultures aspects of HIV as a sexually transmitted infection. This contribution aims to explore the complexity of the symbol of "live sex" (sex without a condom) as an endorsement of risky sexual behaviour.

Methods This is a personal ethnographic account of the discourse surrounding HIV and sexual practise amongst young educated Ugandans.

Results HIV has become embedded into the discourse surrounding sexual practise of young educated professionals in Uganda. Traditions of polygamy in East Africa battle with strong Christian morals. HIV has become inextricably linked to this discussion. It is not only seen as a sexually transmitted infection, but as an integral part of decisions regarding sexual practise. I found two common perspectives. Firstly, participating in "live sex" is used as a deep symbol of trust between partners when embarking on a new monogamous relationship. Secondly, "live sex" is a symbol of romance, of natural pleasure, and a reflection of one's true masculinity. A consistent opinion was; "if you are going to catch it, at least catch it in a moment of ecstasy".

Conclusion For young educated Ugandans, HIV is not just a risk associated with unprotected sexual intercourse, but it has developed additional symbolic meaning to sexual relationships. Despite high levels of understanding regarding HIV and its mode of transmission, educated individuals still engage in risky behaviour. Does this undermine our focus on education in terms of combating spread? This contribution suggests we need to understand more about HIV as a symbol in a complex social and cultural context, and not just as a medically-defined disease.

\section{P4.149 ANTIRETROVIRAL THERAPY AS A RISK FACTOR FOR DEPRESSION, SUICIDAL IDEATION, AND SUICIDE ATTEMPTS AMONG PEOPLE LIVING WITH HIV/AIDS IN THE KATHMANDU VALLEY, NEPAL}

doi:10.1136/sextrans-2013-051184.1044

${ }^{1}$ R M Amiya, ${ }^{2} \mathrm{~K}$ C Poudel, ${ }^{3} \mathrm{~K}$ Poudel-Tandukar, ${ }^{4} \mathrm{~J}$ Kobayashi, ${ }^{1} \mathrm{M}$ Jimba. ${ }^{1}$ University of Tokyo, Tokyo, Japan; 'University of Massachusetts Amherst, Amherst, MA, United States; ${ }^{3}$ Waseda University, Tokyo, Japan; ${ }^{4}$ National Center for Global Health and Medicine, Tokyo, Japan

Background Although antiretroviral therapy (ART) has significantly slowed disease progression and increased longevity among people living with HIV/AIDS (PLWHA), the potential impact on psychological variables is yet poorly understood. Profound changes in the lived experience and perception of illness resulting from ART introduction could potentially influence development of depression, an especially common (25-40\%) and consequential condition in PLWHA. Left untreated, depression contributes to treatment nonadherence and poorer clinical outcomes. This study thus sought to measure the association of ART with depressive symptoms among PLWHA in the Kathmandu Valley, Nepal.

Methods In this cross-sectional study, we surveyed a communitybased sample of 321 PLWHA residing in the Kathmandu Valley, Nepal, using a structured, pre-tested Nepali language questionnaire with face-to-face interviews. The 21-item Beck Depression Inventory (BDI), Nepali version, was used to assess depression in participants. Data were analysed using multiple logistic regression models to examine factors associated with depression, suicidal ideation, and history of suicide attempts, adjusting for potential confounders.

Results Overall, $26 \%$ of participants had depressive symptoms, $14 \%$ reported suicidal thoughts or wishes, and $17 \%$ had ever attempted 